EXTENDED REPORT

\title{
Sports activities 5 years after total knee or hip arthroplasty: the Ulm Osteoarthritis Study
}

K Huch, K A C Müller, T Stürmer, H Brenner, W Puhl, K-P Günther

See end of article for authors' affiliations

Correspondence to:

Correspondence to:
Dr K Huch, Department of Orthopaedic Surgery,

University of Ulm, Oberer

Eselsberg 45, $89081 \mathrm{Ulm}$, Germany; klaus.huch@

rku.de

Accepted 18 April 2005

Published Online First

20 April 2005

\begin{abstract}
Objective: To analyse sports activities of patients with hip or knee osteoarthritis (OA) over lifetime, preoperatively, and 5 years after arthroplasty.

Methods: In a longitudinal four centre study, 809 consecutive patients with advanced OA of the hip (420) or the knee (389) joint under the age of 76 years who required total joint replacement were recruited. A completed questionnaire about sports activities at 5 year follow up was received from $636(79 \%)$ of the 809 patients.

Results: Although most patients with hip (97\%) and knee (94\%) OA had performed sports activities during their life, only $36 \%$ (hip patients) and $42 \%$ (knee patients) had maintained sports activities at the time of surgery. Five years postoperatively, the proportion of patients performing sports activities increased to $52 \%$ among patients with hip OA, but further declined to $34 \%$ among those with knee OA. Accordingly, the proportion of patients with hip OA performing sports activities for more than 2 hours a week increased from 8 to $14 \%$, whereas this proportion decreased from 12 to $5 \%$ among patients with knee OA. Pain in the replaced joint was reported by $9 \%$ of patients with hip and by $>16 \%$ with knee OA.

Conclusion: Differences in pain 5 years after joint replacement may explain some of the difference of sports activities between patients with hip and knee OA. Reasons for reduction of sports activities may include the increasing age of the patients, their worries about an "artificial joint", and the advice of their surgeon to be cautious.
\end{abstract}

A $\mathrm{n}$ increased life expectancy combined with good general health can enable the elderly to participate in diverse sports activities. Osteoarthritis (OA) of the hip or knee can significantly impair the function of the joint, but arthroplasty helps to restore the function and reduce the associated pain. Both should allow a return to sports activities after arthroplasty.

Expert opinions about recommended or allowed sports activities after total joint replacement remain diverse, ${ }^{1}$ and little is known about sports activities actually performed by patients with total joint replacement. In the 1980s, Ritter and Meding observed a significant decrease in several activities except bicycling within 3 years after arthroplasty and recommended the "intelligent participation" in, for example, walking, golf, and bowling, to avoid harm to the prosthesis. ${ }^{2}$ Bradbury et al reviewed 160 patients, including only 56 patients who participated in regular exercise in the year before surgery, and found that 5 years after total knee replacement $43(77 \%)$ had returned to sports. ${ }^{3}$ Of the patients not regularly involved in sports in the year before surgery, only eight patients took up sports after surgery. The three most popular sports in their study were golf $(>50 \%$ preoperatively), bowling ( $>30 \%)$, and tennis $(30 \%)$.

However, the level of sports activities declines with age, as shown by Zahiri et al. ${ }^{4}$ Their patients aged $<60$ years were $30 \%$ more active, on average, than those aged $\geqslant 60$. Therefore, the improved function through total joint replacement in one joint might be antagonised over time by other age related impairments, such as OA in other joints or other comorbidity.

Several specific differences are known for knee and hip joints. Norman-Taylor et al demonstrated that quality of life scores before knee arthroplasty surgery are significantly lower than those before hip joint replacement. ${ }^{5}$ Accordingly, the history of preoperative pain is longer for patients with OA of their knee (about 10 years) than for patients with OA of their hip (about 5 years), ${ }^{6}$ and the outcome for hip joint replacement seems to be significantly better than that for the knee joint. ${ }^{7}$

Our study aimed at analysing and comparing lifetime sports activities of patients with hip or knee OA with the activities performed preoperatively and 5 years after arthroplasty in a large sample of patients undergoing hip or knee replacement due to OA.

\section{MATERIALS AND METHODS}

\section{Study design}

In the Ulm Osteoarthritis Study, a longitudinal four centre study, 809 patients who underwent total joint replacement due to advanced OA of the hip $(n=420)$ or the knee joint $(n=389)$ under the age of 76 years were consecutively recruited between January 1995 and December 1996. Details of the study design have been reported previously. ${ }^{6}$

After giving their written informed consent, patients were interviewed and examined by trained physicians on the day before surgery according to the protocol approved by the ethics committee of the University of Ulm. The standardised investigation included the following baseline data: demographic information, duration and severity of symptoms, drug use, previous medical history.

Between 1 July 2000 and 30 June 2001 follow up information was obtained by a standardised mailed questionnaire from $636(79 \%)$ of the 809 patients at baseline. Of the 809 recruited patients at baseline, 56 (7\%) did not respond (after several initiatives), 56 (7\%) refused to be involved further in the study, 44 (5\%) had died, and $17(2 \%)$ could not be contacted because their address was unknown.

\section{Questionnaire}

Participation in the following sports was ascertained in both the baseline and the follow up questionnaire: hiking, riding, dancing (including ballet dancing and jazz dancing), aerobics, swimming/aqua jogging, soccer/handball/volleyball/basketball, 


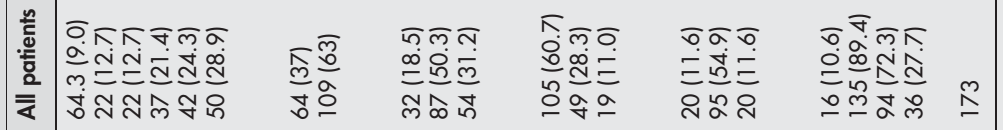

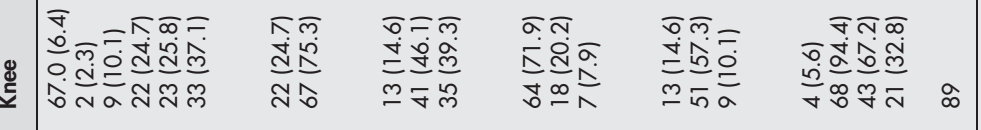

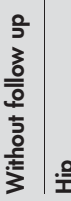

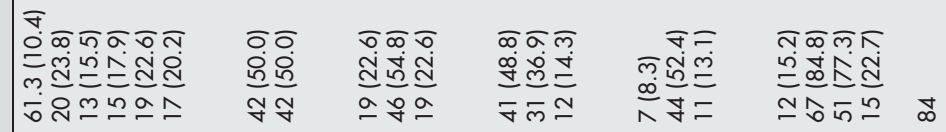

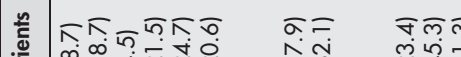

船

$\overline{\bar{\varepsilon}}$

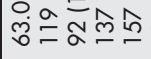

लें ชู

ขึ่

ำ

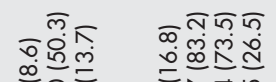

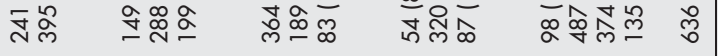

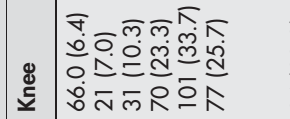

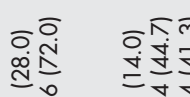

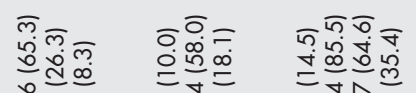

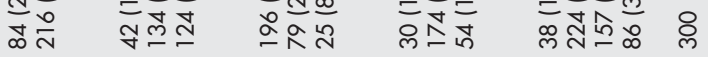

\begin{tabular}{l}
3 \\
3 \\
$\frac{0}{0}$ \\
\hline
\end{tabular}

๘๐ニลス=

a 2000.0

웡

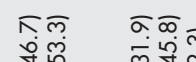

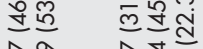

ठำ

$=\stackrel{\bar{n}}{=}$

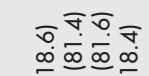

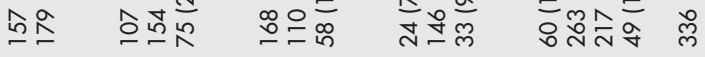

क

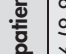

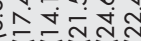

年

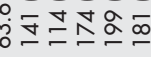

रुल चซल

लिं तुำ

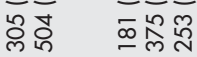

वृ

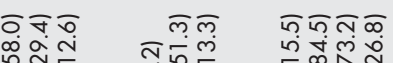

a

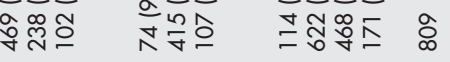

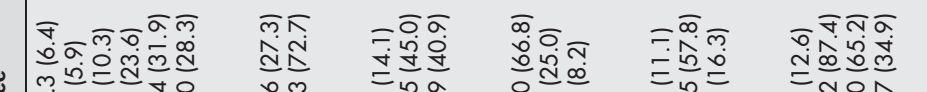

\&

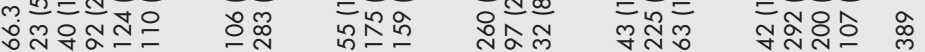

ํำ

:

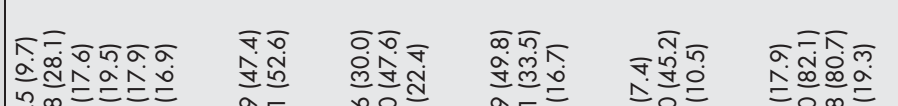

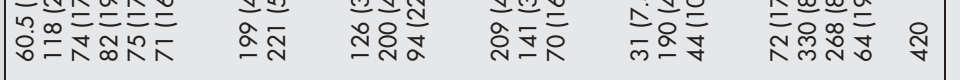

$\stackrel{\oplus}{\frac{1}{ \pm}}$

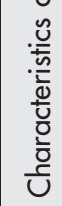

高 -4 -

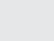


down hill skiing, cross country skiing/jogging, boxing, wrestling. In addition, patients could name other activities in a free text area and commonly named golfing, bowling, tennis, which were included in this analysis.

In the baseline questionnaire we asked for sports performed during a patient's lifetime and those pursued until total joint replacement. In the 5 year follow up we inquired about the current level of sports activities. On both occasions we ascertained whether patients took part in sports activities for one or more hours a week.

The patients had the opportunity to give the following explanations for a reduction of their sporting activities: pain in the replaced joint, pain in other regions, as a precaution, to go easy on the artificial joint, others (including free text comment-for example, owing to age, impossible to perform, heart failure, instability of the joint, vertebral pain).

The patients were also interviewed for comorbidity (for example, diabetes, hypertension, cerebral infarction, fractures, myocardial infarction, cancer, thromboembolism, gastrointestinal ulcers, or spinal disorders) and for surgical procedures in other major joints of the lower extremities. They also had the opportunity to mark painful joints on a diagram of the whole body.

In the baseline questionnaire, the distribution of radiographically determined OA in hip, knee, and finger/carpal joints (grade $\geqslant 2$ according to Kellgren and Lawrence ${ }^{8}$ ) was documented to classify mono- or bilateral OA and mono- and polyarticular distributions. The method for identification of polyarticular disease has been described earlier. ${ }^{6}$ A definition of generalised OA required the involvement of at least two distal or proximal interphalangeal joints and at least one carpometacarpal joint in addition to knee or hip OA. ${ }^{6}$

\section{Data analysis}

Participation in sports activities at various points of time was primarily analysed by descriptive statistics. In addition, multiple logistic regression was carried out to assess determinants of participation in sports activities for one or more hours per week 5 years after total knee or hip arthroplasty. Statistical analyses were performed using Statistical Analysis Systems (version 6.10), SAS Institute, Cary, North Carolina.

\section{RESULTS}

Overall, 809 patients receiving a total joint replacement of their knee or hip were recruited (table 1).

As table 1 shows, the distribution of major sociodemographic characteristics was very similar among all patients recruited and among these who participated in the 5 year follow up. Mean age at baseline was about 63 years. The female to male ratio was about $2: 1$. More than $75 \%$ of the patients were either overweight or obese. About $40 \%$ had ever smoked, but only a minority of about $13 \%$ were still smoking at the time of recruitment. Over $50 \%$ of the patients were hypertensive. The great majority of participants (300/389 $(77 \%)$ of the participants with knee OA and $336 / 420(80 \%)$ of the participants with hip OA) could be followed up 5 years later. As expected, the loss of patients for follow up was age dependent with $28.9 \%$ of those lost aged over 70 years at baseline compared with $21.4 \%$ in the whole cohort.

The distribution of unilateral and bilateral (around 84\%) $\mathrm{OA}$ as well as the distribution of mono-/biarticular and polyarticular (around 27\%) OA were similar at baseline and at the 5 year follow up (table 1).

Five years after total joint replacement an age dependent decline in participation in sports activities for one or more hours a week could be seen, especially for patients with knee OA (fig l).

Men were more active than women at lifetime activities (79\% $v 64 \%$ with one or more hours of sports activities a week) and at activities performed 5 years after total joint replacement ( $38 \% \vee 23 \%)$.

Although most patients with hip (97\%) and knee (94\%) OA had performed sports activities during their life, only $36 \%$ (patients with hip OA) and $42 \%$ (patients with knee OA) had maintained sports activities at the time of surgery (fig 2). Five years postoperatively, the proportion of patients performing sports activities increased to $52 \%$ among patients with hip OA, but further declined to $34 \%$ among patients with knee OA. Accordingly, the proportion of patients with hip OA performing sports activities for more than 2 hours a week increased from 8 to $14 \%$, whereas this proportion further decreased from 12 to $5 \%$ among patients with knee OA (fig 2). Overall, more active patients with a higher intensity of sports activities could be seen after hip replacement than after knee replacement (fig 2).

Figure 3 demonstrates that biking, hiking, and swimming were the most important lifetime sports activities among patients undergoing hip or knee replacement. However, preoperatively only a few patients (more in the knee than in the hip group) were still able to continue these activities, but most patients (more in the hip than in the knee group) were able return to these activities after surgery and maintained their activity at the 5 year follow up (fig 3). Also for other sports activities like skiing, dancing, and gymnastics, patients with hip OA improved more after surgery than patients with knee OA (fig 3).

Pain in the replaced joint was referred to as a reason for the reduction of sports activities after total joint replacement
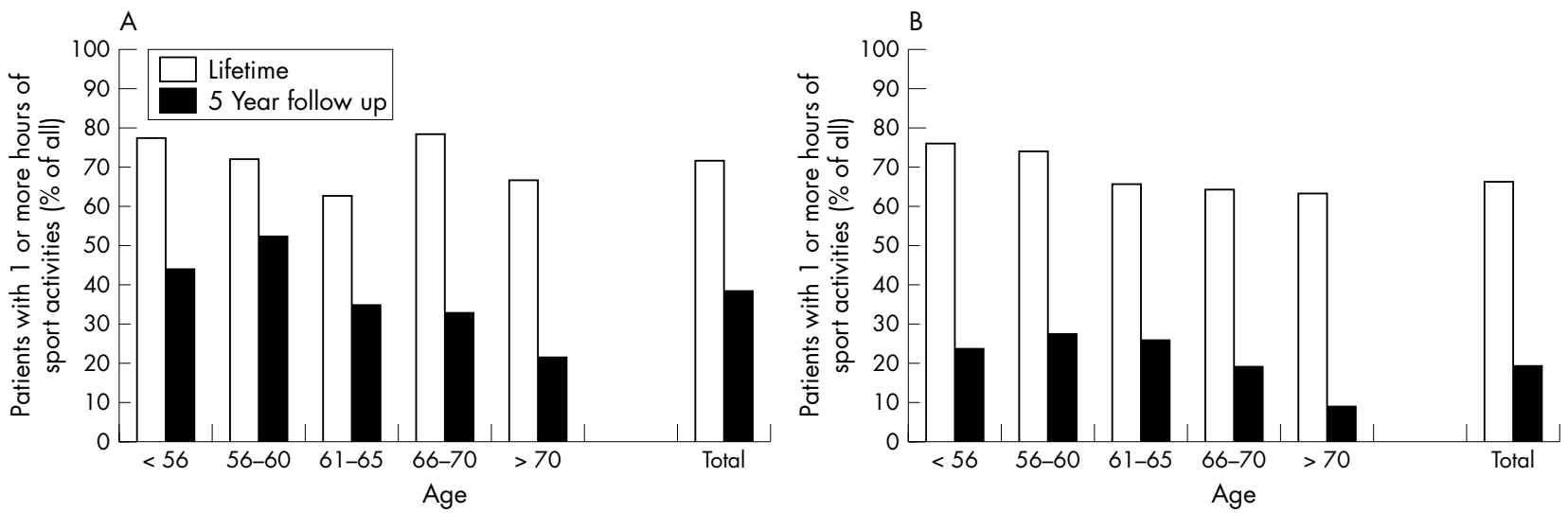

Figure 1 Percentage of patients with hip (A) and knee (B) OA performing sport for one or more hours a week according to age, before and 5 years after total joint replacement. 

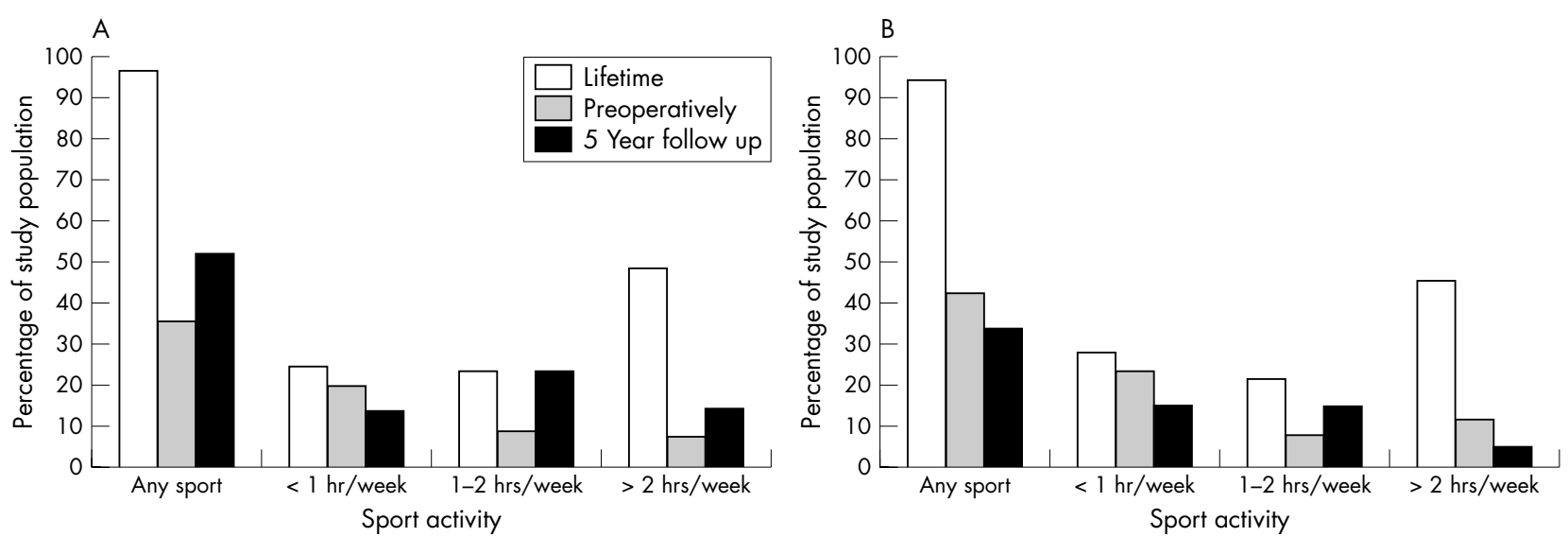

Figure 2 Percentage of patients with hip (A) and knee (B) OA performing sport during lifetime, preoperatively, and at the 5 year follow up at different intensities.

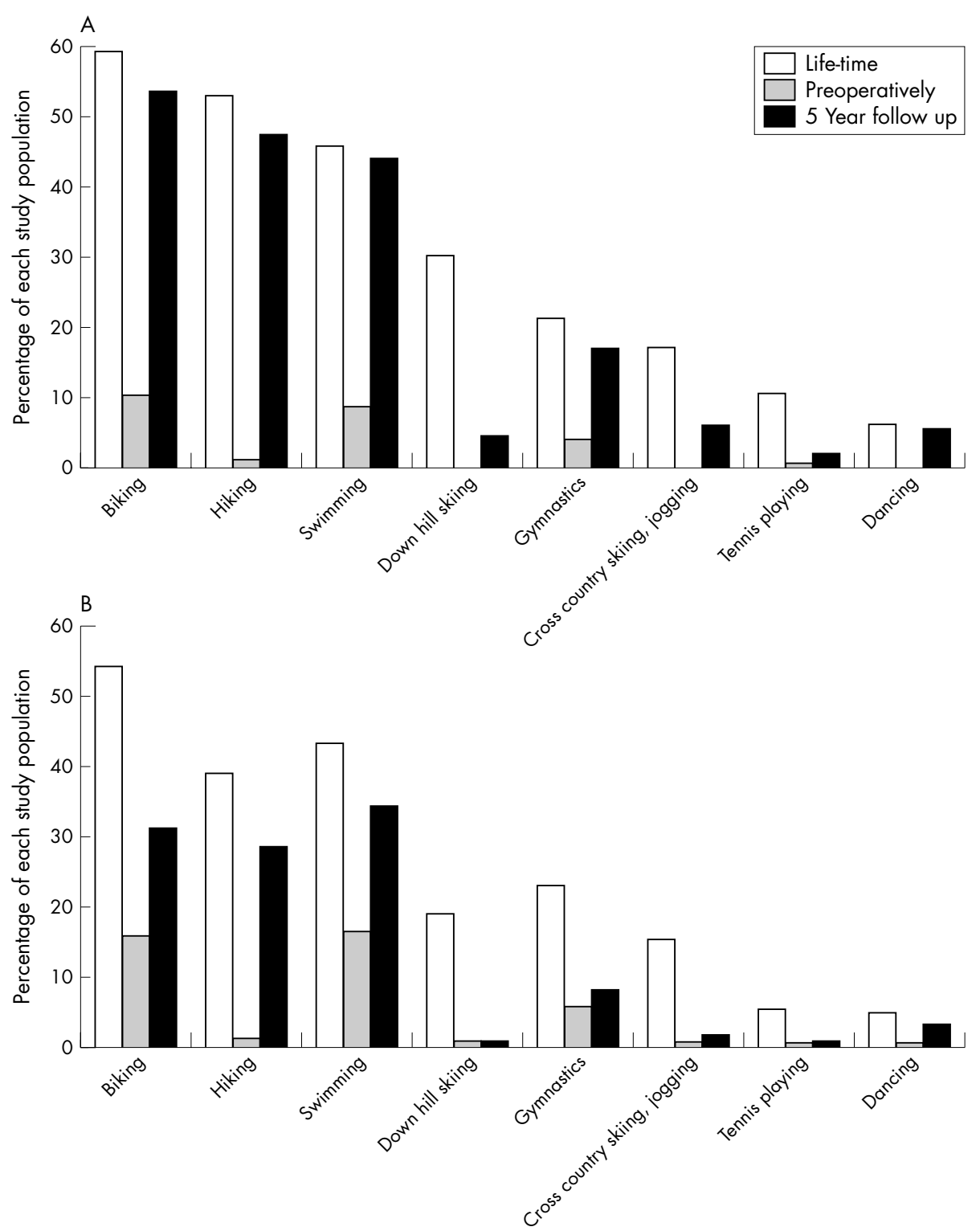

Figure 3 Comparison of specific sports activities at three different times (lifetime, preoperatively, and at 5 year follow up for patients with hip (A) and knee (B) OA. 
Table 2 Main reasons for the reduction of sports activities 5 years after hip or knee replacement

\begin{tabular}{lcll}
\hline Reasons & $\begin{array}{l}\text { Hip } \\
(\mathbf{n}=\mathbf{2 8 5})\end{array}$ & $\begin{array}{l}\text { Knee } \\
(\mathbf{n}=\mathbf{3 1 2})\end{array}$ & $\begin{array}{l}\text { Total } \\
(\mathbf{n}=597)\end{array}$ \\
\hline Pain replaced joint & 8.8 & 16.3 & 12.7 \\
Pain elsewhere & 25.6 & 29.2 & 27.5 \\
Precaution & 53 & 41.7 & 47.1 \\
Others & 11.9 & 10.6 & 11.2 \\
No statement & 0.7 & 2.2 & 1.5 \\
\hline \multicolumn{2}{l}{} \\
\multicolumn{2}{l}{ Results shown as percentages. } \\
\hline
\end{tabular}

about twice as often for the knee as for the hip joint, whereas precaution was more often mentioned for the hip than for the knee joint (table 2).

In a multivariable analysis, the following factors at baseline were associated with participation in sports activities for one or more hours a week 5 years after total knee or hip arthroplasty: age (odds ratio (OR), 95\% confidence intervals (95\% CI), for age groups 61-65, 66-70, and 71-75 compared with age group $\leqslant 60: 0.65,0.34$ to $1.26 ; 0.67,0.34$ to 1.30 ; and $0.37,0.17$ to 0.77 , respectively), sex (for men compared with women: 1.44, 0.86 to 2.41), replaced joint (for hip versus knee: $2.58,1.49$ to 4.47$)$, heavy physical work $(0.47,0.26$ to $0.83)$, smoking $(0.31,0.14$ to 0.66$)$, sports activities (before replacement $2.80,1.51$ to 5.18 ; lifetime sports activities 2.54, 1.43 to 4.52 ).

\section{DISCUSSION}

To our knowledge, this multicentre study performed in four institutions is the first large scale epidemiological cohort study comparing participation in sports activity performed during lifetime, directly before, and 5 years after surgery among patients undergoing primary total joint replacement for hip or knee OA.

At baseline, the two groups of patients with hip/knee OA differed in the following variables: ${ }^{6} 47 / 27 \%$ men, $60.5 /$ 66.3 years old, 69.9/73.4 for pain on a visual analogue scale (ranging from 0 (no pain) to 100 ), 82.1/87.4\% bilateral OA, $19.3 / 34.9 \%$ generalised OA. These differences might, at least partly, be responsible for differences in postoperative sports activities in both cohorts.

Five years after total joint replacement of the hip or knee joint, many patients had regained some of their lifetime sports activities. This was especially true for biking, hiking, and swimming. Patients with hip OA were particularly more active in biking, hiking, swimming, down hill skiing, gymnastics, and cross country skiing/jogging than patients with knee OA at the 5 year follow up (fig 3 ).

Brander et al did not compare differences in sports activities after total joint replacement in hip and knee joints, ${ }^{9}$ but they analysed the functional outcome of 80 people aged 80 and older (average 82.7 years for total knee and 82.6 for total hip arthroplasty), an age spectrum not included in our study. The mean of the Harris Hip Score improved from 60 to 88, whereas the mean of the Hospital for Special Surgery Score (knee) changed from 55 to 80.5. For the patients with hip/knee OA the preoperative pain was severe to moderate in $72 \% / 93 \%$ of the patients, the postoperative pain was mild to none in $100 \% / 98 \%$. The ability of patients with hip/knee OA to walk more than five blocks increased from $7 \% / 2 \%$ to $54 /$ $50 \%$. The study demonstrated not only the successful functional outcome of arthroplasty in the elderly but also (slightly) better functional values for patients with hip OA.

However, Weiss et al demonstrated for patients with total knee replacement that kneeling and gardening were not only among the three most important activities mentioned by patients but also among the three most difficult ones. ${ }^{10}$ This indicates that function might be limited after total joint replacement.

Especially in the lower extremity, the durability of total joint replacement seems to be dependent on mechanical forces. Kuster et al estimated the maximum tibiofemoral compressive force from kinematic and kinetic measures obtained during level (about 3.9 times body weight during the early stance phase) and down hill walking (about eight times body weight). ${ }^{11}$ They also evaluated three different knee endoprosthesis designs for loads estimated for cycling, power walking, hiking, and jogging and concluded that jogging or running should be discouraged after knee arthroplasty. ${ }^{12}$

This might explain why surgeons give different recommendations about activities after arthroplasty. Patients, on the other hand, do not always take notice of the recommendations of their surgeons. Mont et al examined 50 male and 8 female tennis players (mean age 70 years) after total hip arthroplasty. ${ }^{13}$ Although only $14 \%$ of the patients' surgeons approved this activity postoperatively, all patients played tennis three times a week on average 1 year after arthroplasty. Three patients $(5 \%)$ required revision after a mean of 8 years.

Schmalzried et al analysed the activity of 111 nonrandomised patients with either knee or hip replacement using an electronic pedometer. ${ }^{14}$ The most active patient walked more than 3.5 times the average number of steps per day. Patients under 60 years of age walked 30\% more than those older than 60 . As in our study, men were more active then women and walked $28 \%$ further. The authors postulate that these differences between patients might influence the survival of knee or hip arthroplasties.

Gschwend et al examined differences in aseptic loosening between an active (alpine and cross country skiing) (mean age 65 years, range 47-84) and an inactive (mean age 65 years, range 42-79) group of 50 patients each for total hip replacement and could not find a negative effect of skiing on the artificial components after 5-10 years of follow up. ${ }^{15}$ After an average follow up of 5.8 years, Dubs et al ${ }^{16}$ observed a revision rate of $1.6 \%$ in the 61 sporty patients after total hip replacement, whereas the 49 patients without sports activities had a revision rate of $14.3 \%$. Similar results were described for hip joints by von Strempel et al, with a loosening rate of $5 \%$ in the sports group and $10 \%$ in the no-sports group, ${ }^{17}$ and by Widhalm et al, with loosening in $18 \%$ versus $57 \% .^{18}$

However, Kilgus et al ${ }^{19}$ found a $6 \%$ revision rate for less active patients and a $28 \%$ revision rate for active patients with conventional cemented stemmed prosthesis. They also described a higher revision rate for patients without OA than for patients with OA as a cause for total hip replacement (41 failures per 1000 person-years of follow up versus 36 for the active and 16 versus 4 for the less active group) and discussed a reduced bone quality in the former group as a possible explanation for this observation.

Healy et al describe in their review the technical advances that have reduced the loosening of implants in young active patients. ${ }^{1}$ For this group they recommend cementless acetabular implants in hip arthroplasty and cemented components in knee and shoulder replacement.

Among the patients included in this study, moderate activity was generally allowed, but we advised against high impact sports activities. Owing to missing detailed recommendations about sports activities after total joint replacement in our study protocol and owing to the four centre design of our study, slightly different suggestions are possible in different institutions.

Age above 75 years was an exclusion criterion in our study. Therefore, our results might not be representative for the elderly. 
Because we did not include an age matched control group without severe OA of the hip or knee in our study, we cannot estimate the number of patients who have reduced their activities owing to their increasing age rather than their arthroplasty. However, we did observe an age correlation of sports activities in our cohort.

Only $14 \%$ of our study group did not respond to our 5 year follow up questionnaire or refused to participate. Reasons for rejection included poor general health status. Nonresponders were older and likely to be less active than the responders, which may have led to a slight overestimation of the proportion of active patients at the 5 year follow up.

In general, total joint replacement allows a significant increase of sports activities at a 5 year follow up. In our study, patients with hip OA had significantly better results than patients with knee OA. One reason for this difference might be that about $9 \%$ of the patients with hip OA, but over $16 \%$ of those with knee OA reported pain in their replaced joint. The patients with hip OA were also characterised by a higher proportion of men and younger patients (who are more likely to maintain sports activities according to our findings), a more localised pattern of OA distribution, and a lower pain score than the patients with knee OA. Nevertheless, a much higher participation in sports activities was seen in patients with hip OA than in knee OA, even after control of potential confounders (including age) in multivariate analysis.

General reasons for a reduction of (special) sports activities after total joint replacement may be the increasing age of the patients, worries connected with an "artificial joint", and the advice of the surgeon or physician to be cautious.

The possible influence of sports activities on the failure of total joint replacements has to be evaluated in a long term follow up of our patients.

At the moment we generally suggest moderate activity, but we advise against high impact sports activities (with often uncontrolled and physically powerful joint movements-for example, soccer, down hill skiing, tennis) after total joint replacement.

\section{ACKNOWLEDGEMENTS}

We thank the Department of Trauma Surgery, University of Ulm (Chairman: Professor Dr L Kinzl); the Department of Orthopaedic Surgery, Klinik Dr Baumann, Stuttgart (Professor Dr CT Trepte); and the Department of Orthopaedic Surgery, Hessing-Klinik Augsburg (PD Dr T Naumann) for their collaboration in patient recruitment. We also thank Dr E Raum for valuable contributions to data analysis. This work was supported by the Federal Ministry of Research and Technology (BMFT, 01 EF 9406).

\footnotetext{
Authors' affiliations

K Huch, K A C Müller, W Puhl, Department of Orthopaedics, University of Ulm, Oberer Eselsberg 45, 89081 Ulm, Germany
}

T Stürmer, Division of Pharmacoepidemiology and Pharmacoeconomics, Division of Preventive Medicine, Brigham and Women's Hospital, Harvard Medical School, 1620 Tremont Street, Suite 3030, Boston, MA 02120, USA

H Brenner, Department of Epidemiology, German Centre for Research in Aging (DZFA), Bergheimer Str 20, 69115 Heidelberg, Germany K-P Günther, Department of Orthopaedics, University of Dresden, Fetscherstr 74, 01307 Dresden, Germany

\section{REFERENCES}

1 Healy WL, lorio R, Lemos MJ. Athletic activity after joint replacement. Am J Sports Med 2001;29:377-87.

2 Ritter MA, Meding JB. Total hip arthroplasty. Can the patient play sports again? Orthopedics 1987; 10:1447-52.

3 Bradbury N, Borton D, Spoo G, Cross MJ. Participation in sports after total knee replacement. Am J Sports Med 1998;26:530-5.

4 Zahiri CA, Schmalzried TP, Szuszczewicz ES, Amstutz HC. Assessing activity in joint replacement patients. J Arthroplasty 1998;13:890-5.

5 Norman-Taylor FH, Palmer CR, Villar RN. Quality-of-life improvement compared after hip and knee replacement. J Bone Joint Surg $\mathrm{Br}$ 1996;78:74-7.

6 Günther KP, Stürmer T, Sauerland S, Zeissig I, Sun Y, Kessler S, et al. Prevalence of generalised osteoarthritis in patients with advanced hip and knee osteoarthritis: the Ulm Osteoarthritis Study. Ann Rheum Dis 1998;57:717-23

7 Bachmeier CJ, March LM, Cross MJ, Lapsley HM, Tribe KL, Courtenay BG, et al. A comparison of outcomes in osteoarthritis patients undergoing total hip and knee replacement surgery. Osteoarthritis Cartilage 2001;9:137-46.

8 Kellgren JH, Lawrence JS. Atlas of standard radiographs of arthritis. Oxford: Blackwell Scientific Publications, 1963

9 Brander VA, Malhotra S, Jet J, Heinemann AW, Stulberg SD. Outcome of hip and knee arthroplasty in persons aged 80 years and older. Clin Orthop 1997; 345:67-78

10 Weiss JM, Noble PC, Conditt MA, Kohl HW, Roberts S, Cook KF, et al. What functional activities are important to patients with knee replacements? Clin Orthop 2002:172-88.

11 Kuster MS, Wood GA, Stachowiak GW, Gächtner A. Joint load considerations in total knee replacement. J Bone Joint Surg Br 1997;79:109-13

12 Kuster MS, Spalinger E, Blanksby BA, Gachter A. Endurance sports after total knee replacement: a biomechanical investigation. Med Sci Sports Exerc 2000;32:721-4.

13 Mont MA, Laporte DM, Mullick TM, Silberstein CE, Hungerford DS. Tennis after total hip arthroplasty. Am J Sports Med 1999;27:60-4.

14 Schmalzried TP, Szuszczewicz ES, Northfield MR, Akizuki KH, Frankel RE, Belcher $G$, et al. Quantitative assessment of walking activity after total hip or knee replacement. J Bone Joint Surg Am 1998;80:54-8.

15 Gschwend N, Frei T, Morscher E, Nigg B, Löhr J. Alpine and cross-country skiing after total hip replacement 2 cohorts of 50 patients each, one active, the other inactive in skiing, followed for 5-10 years. Acta Orthop Scand 2000;71:243-9.

16 Dubs L, Gschwend N, Munzinger U. Sport After total hip arthroplasty. Arch Orthop Trauma Surg 1983;101:161-9.

17 Von Strempel A, Menke W, Wirth CJ. Sportliche Aktivitäten von Patienten mit zementfrei implantiertem Hüftgelenkersatz. Prakt Sport-Traum Sportmed 1992;2:58-64.

18 Widhalm R, Höfer G, Krugluger J, Bartalsky I. [ls there greater danger of sports injury or osteoporosis caused by inactivity in patients with hip prosthesis? Sequelae for long-term stability of prosthesis anchorage. ] Z Orthop, 1990; 128:139-43.

19 Kilgus DJ, Dorey FJ, Finerman GAM, Amstutz HC. Patient activity, sports participation, and impact loading on the durability of cemented total hip replacements. Clin Orthop 1991;269:25-9. 